\title{
Testing an Arduino-Based Approach for Full-Duplex Voice Communication and Body-Parameter Sensing Electronics for Use with Smart Clothing
}

\author{
Miroslav Joler (D), Andrej Berkarić, and Valentin Klen \\ Faculty of Engineering, University of Rijeka, Rijeka HR-51000, Croatia \\ Correspondence should be addressed to Miroslav Joler; mjoler@riteh.hr
}

Received 27 December 2018; Revised 24 March 2019; Accepted 22 April 2019; Published 8 May 2019

Academic Editor: N. Nasimuddin

Copyright (c) 2019 Miroslav Joler et al. This is an open access article distributed under the Creative Commons Attribution License, which permits unrestricted use, distribution, and reproduction in any medium, provided the original work is properly cited.

In this paper, we discuss the state of the art and trends in the field of smart clothing development and show two circuits that we assembled in search for adequate solutions. The circuits are based on Arduino concept and achieve a full-duplex voice communication and real-time publishing of temperature measurement to a designated web page. The characteristics of the two circuits are discussed as well as the prospects for their future improvements.

\section{Introduction}

Development of communication networks and microelectronics is bringing us opportunities to work towards development of smart clothing. Garment considered as smart clothing is envisioned to be equipped with various wireless sensors and communication capabilities towards remote entities. Sensors should enable either measurement of body parameters such as the heart rate $[1,2]$, blood pressure, glucose level [3,4], or air quality [5] and wirelessly transmit the measured data to some main control unit (MCU) that is also being worn on a body or being outside it such as a mobile phone, which would then display them and/or store them, make further calculations using them, and alert the wearer and other interested parties about possibly concerning values of some body parameters, or forward them to a remote center, such as a cloud storage. Communication links should provide reliable and secure data transmission from the sensors to MCU in diverse environments and under different body motions and provide a range from a few tens of centimeters to several meters or tens of meters, depending on the scenario of the particular application.

The integration of the sensors and communication chips into the clothing should satisfy the requirements of being mechanically robust against various natural strains during body movement, being noninvasive to make wearing the clothing comfortable enough, be resistant to penetration of moisture, be water repellent, be either washable or easy to remove and mount before and after washing, respectively, have a good thermal stability, and, depending on the application, have a power supply secured over a long enough period of time. Sensors measuring body parameters and transferring that information to a mobile phone have recently appeared on the market in the form of watches equipped with a few sensors that typically monitor heart rate and track the physical activity by means of the number of steps walked, possibly including GPS-acquired locations, calories burned, and sleep cycles, for example. In the near future, clinicalaccuracy blood pressure measurement is expected to become available on a wrist watch as well [6]. Succeeding that, we can expect it to be integrated with the already present features, for more complete monitoring. Most recent applications transfer sensors data from a wrist watch to a wearer's mobile phone via a Bluetooth connection [7]. Such "fitness tracker" watches are typically powered on the principle of a mobile phone-by a regular periodic charging using a USB cable. However, in spite of their attractiveness, this kind of wearables does not match the notion of smart clothing because the key devices and their functions are placed outside the clothing.

There have also recently appeared specialized pieces of garment in the form of either a special shirt, belt, or socks that should help athletes or recreational players to monitor 
their body parameters during a workout or a game $[8,9]$ and the data are also transmitted to wearer's cellphone and then forwarded to a cloud using a mobile network the phone is connected to. Wearable textile as an input device has also been recently researched $[10,11]$ to enable mobile device control without having to look at it. For example, one recent application [12] has touch sensors sewn into a popular jeans jacket, to enable a user to control his cellphone for popular functions such as control keys of a music player (i.e., next song, previous song, pause, play, etc.), or voice-guided navigation and is also based on the Bluetooth connection to a user's cellphone. True garment-embedded electronics and communication devices that will be able to not only transfer the sensor-taken data, but also enable a user to have a fullduplex voice communication with his peer, without being dependent on the usage of mobile phone and its network or carrying a backpack with a radio device, are yet to be developed for comfortable wearing, dependable and robust operation of both the measurement and communication part. We are interested in achieving garment-embedded solution that will be more comfortable for wearing and maintenance, yet offer embedded communication and physical-parameter sensing features. In this paper, we present our early examination of possible implementations.

Achievements in the area of smart clothing have not been significant so far due to multiple factors: insufficient reliability of components and insufficient air-permeability of garment, high cost of manufacturing of such garment, and being impractical to wash. Besides it, such a garment was pretty rigid and uncomfortable to wear for being based on the e-textiles [13] (i.e., textiles having a property of electric conductivity) and as such impractical for everyday wearing. However, market analyses forecast a steady growth of this segment of the market at an annual rate of $12 \%$, even up to $25 \%$ percent till 2022 [14].

Such a forecast and the technological trends that lead to an extensive and ubiquitous use of the Internet of things, major part of which is wireless sensors, which are anticipated to take a significant part in the realm of upcoming $5 \mathrm{G}$ networks, encourage various research efforts throughout the world that are trying to propose a solution to some of the key challenges in the development of smart clothing, which are (a) embedding wireless sensors: locations, power supply, and data transfer; (b) radio-frequency $(\mathrm{RF})$ transceivers: the size, power consumption, choice of arbitrary frequency, and output power; (c) antennas: placement, size, and the design for an arbitrary frequency; (d) power supply: battery vs. energy harvesting; (e) seamless integration of everything in a noninvasive way [15] while having mechanical robustness, thermal stability, water repellency, or moisture immunity and maintaining or enabling practical washability features [1618]. In addition to the aforementioned features, we also look forward to enabling a full-duplex voice communication [19], which imposes an additional issue of whether using one or two antennas, whether external or on-chip antennas, and how to connect them with transceivers.

As a novelty with respect to a typical approach nowadays, which is based on the Bluetooth connection between the sensors and wearer's cellphone, we are exploring solutions

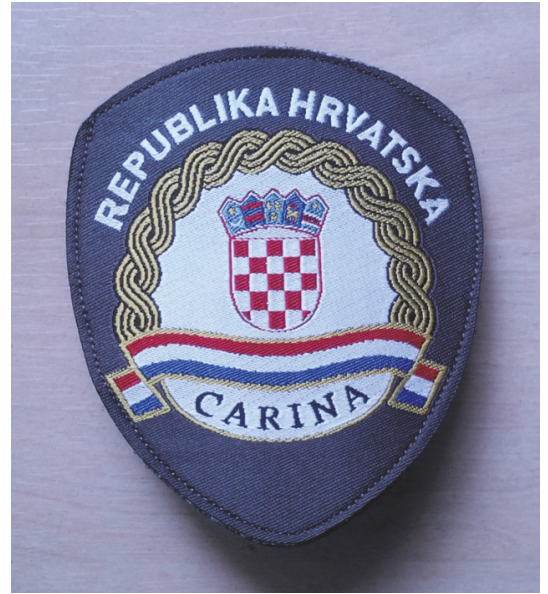

FIGURE 1: A sleeve badge textile antenna proposed in [20].

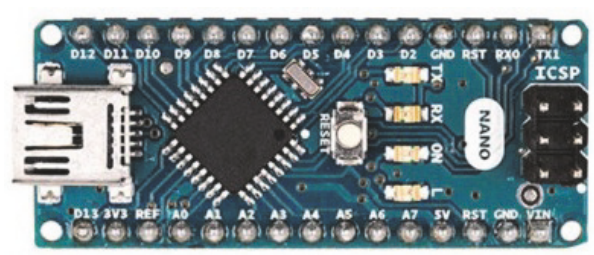

FIgURE 2: An Arduino nano development board.

that will enable cellphone-independent full-duplex voice communication suitable for group-based communications that are found in scenarios involving rescue services (e.g., firefighters or paramedics), military troops, and similar situations. Multiple wearable sensors are foreseen to be integrated via the MCU. In this paper, we explore achieving a full-duplex voice communication and remotely accessible real-time temperature measurement using an Arduino-based MCU.

\section{Arduino-Based Full-Duplex Voice Communication}

Initially, we had proposed [20] a jacket sleeve badge wearable textile antenna (Figure 1), as a basis to develop a complete solution of a smart jacket, which includes RF transceivers, wearable sensors, and renewable power supply by energy harvesting. In this early approach, we chose to examine the Arduino platform [21] as the basis for the communication solution. Arduino is nowadays a popular computing platform that enables programming of hardware using the arduino.ide language, a language similar to $\mathrm{C}$ and $\mathrm{C}++$ programming language. Arduino, as a paradigm, had started an avalanche of small programmable devices around the world, which quickly became popular for its low cost, simplicity, and openness. Arduino and its derivatives now offer a variety of development boards, as the key unit to control various connected peripherals, such as sensors, switches, and indicators. Among them, we chose to use Arduino nano [22] for its small dimensions (Figure 2), while having enough computational 


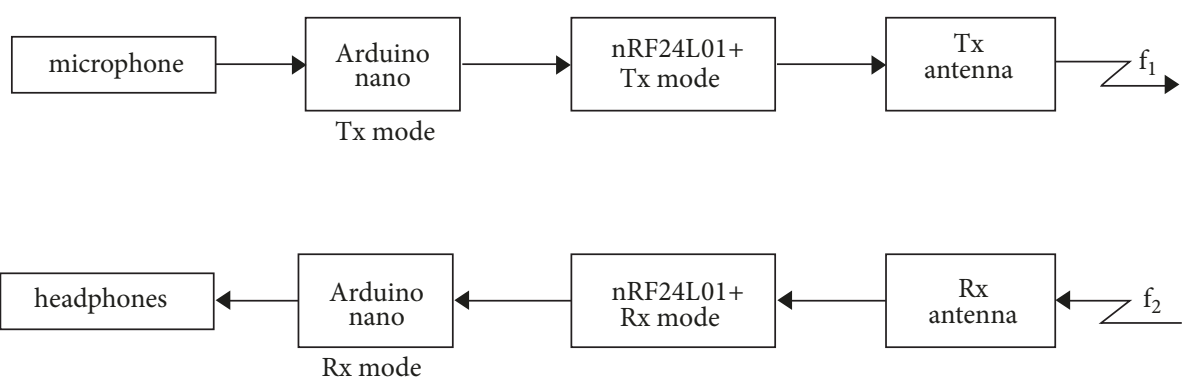

(a) $\mathrm{Tx} / \mathrm{Rx}$ principle schematic

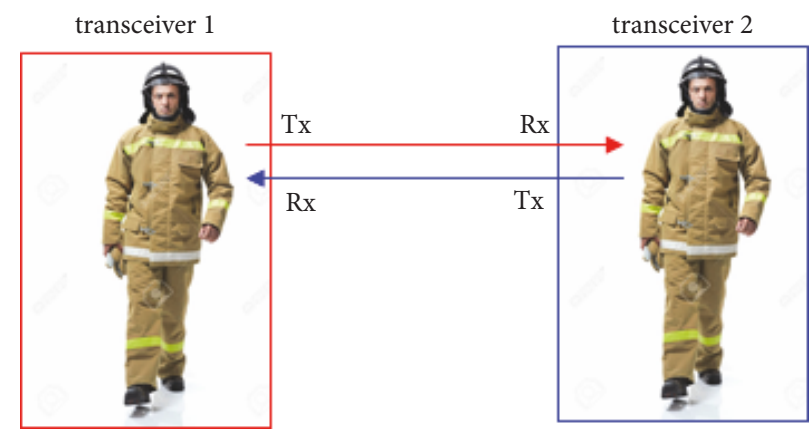

(b) Firefighter scenario

FIGURE 3: Realization of a full-duplex voice link using Arduino nano and nRF24L01+ transceiver: (a) the principle schematic; (b) a possible implementation scenario. Image source for the firefighter: $123 R F$.

and communication capabilities for our scenario. Its size is only $45 \mathrm{~mm}$ x $18 \mathrm{~mm}$, weighs only $7 \mathrm{~g}$, and will here serve as an MCU. As it does not have the RF communication feature built in, the key communication element for that task was chosen to be a popular RF transceiver widely known as nRF24L01 $[23,24]$. Using a low power consumption, it operates with one microcontroller in the 2400..2525 MHz ISM band. To communicate with another microcontroller, it utilizes a serial peripheral interface (SPI) [25], which enables it to work either in the transmit $(\mathrm{Tx})$ or in the receive $(\mathrm{Rx})$ mode at a time. The user can set a desired communication channel frequency, the output power level of $0 \mathrm{dBm},-6 \mathrm{dBm}$, or $-18 \mathrm{dBm}$, and the data rate of either $250 \mathrm{kbps}, 1 \mathrm{Mbps}$, or $2 \mathrm{Mbps}$. There are 124 channels, which are separated $1 \mathrm{MHz}$ at the data rate of $1 \mathrm{Mbps}$ and $2 \mathrm{MHz}$ at the data rate of $2 \mathrm{Mbps}$. The receiver sensitivity is $-82 \mathrm{dBm}$ at $2 \mathrm{Mbps}$ and $-85 \mathrm{dBm}$ at $1 \mathrm{Mbps}$. For the testing purposes, commercial antennas were used instead of our antenna in Figure 1, as we needed more than one antenna in the test assembly.

Since the RF transceiver operates in either Tx or Rx mode via an SPI protocol, to establish a full-duplex wireless link, as illustrated in Figure 3, we had to combine two Arduino nanos, two transceivers, and two antennas at each side, one pair of them to work in the Tx mode and the other to operate in the Rx mode. Figure 4 shows the assembled prototype $\mathrm{Tx} / \mathrm{Rx}$ circuit for the two sides. (Antennas in this assembly were used merely to make initial tests and obviously not planned for a realistic scenario, due to their size and type.) Besides the principle blocks shown in Figure 3(a), there was also a need to secure a stable $3.3 \mathrm{~V}$ bias voltage for

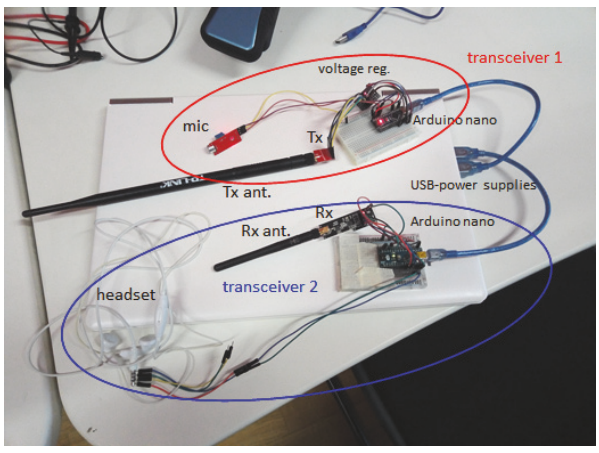

FIGURE 4: An early assembly of a full-duplex voice communication link.

each transceiver. As the respective output pin of Arduino nano does not guarantee a stable $3.3 \mathrm{~V}$ voltage, external voltage regulators were employed for that purpose. In this test, the transceivers Tx 1 and Tx 2 on each side were set to work at $2401 \mathrm{MHz}$ and $2475 \mathrm{MHz}$, respectively. Figure 5 shows a separate measurement of the two signals, which was performed by an Aaronia Spectran HF-6065 V4 spectrum analyzer [26]. Note that besides the two designated signals at $2401 \mathrm{MHz}$ and $2475 \mathrm{MHz}$, the third strong signal at about $2455 \mathrm{MHz}$ is an unrelated signal of a nearby WLAN router.

2.1. Field Testing. In our field tests of this initial assembly, we first achieved clear transmission at the distance of about 5 $\mathrm{m}$, whereas at range beyond $15 \mathrm{~m}$, the reception deteriorated 


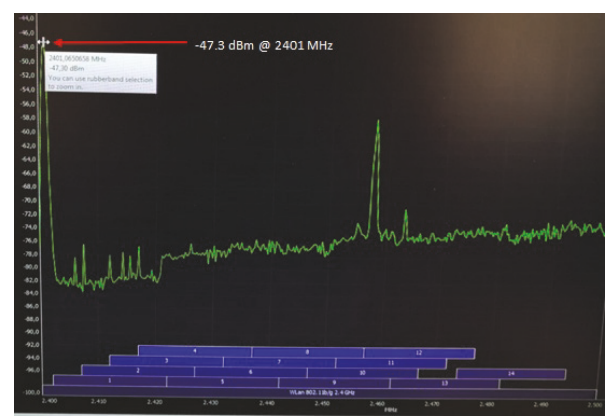

(a) Signal of Tx 1

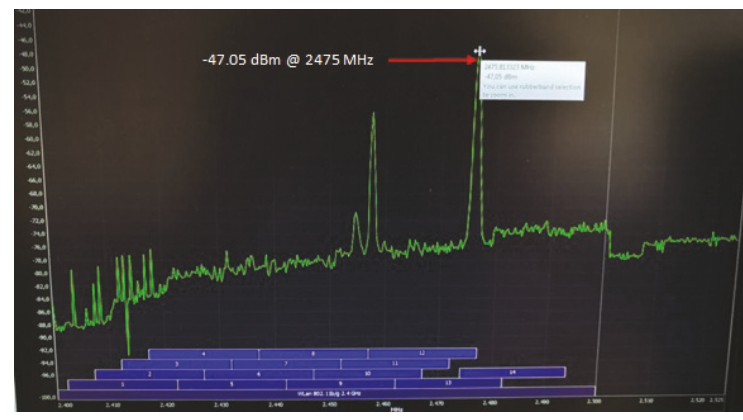

(b) Signal of Tx 2

FIgURE 5: Measurement of the operational frequencies of Tx 1 and Tx 2 by a spectrum analyzer.

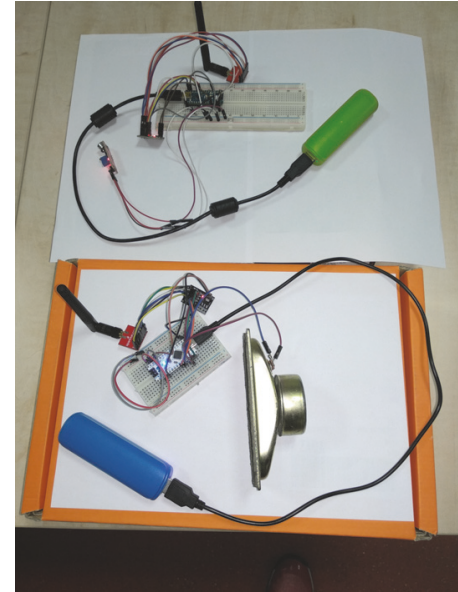

(a) Range-test circuits with higherquality transceivers

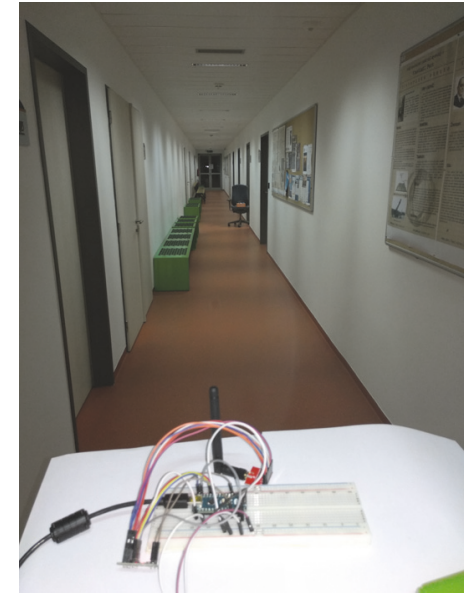

(b) A test at $15 \mathrm{~m}$ range in a school hallway

FIGURE 6: Full-duplex voice communication testing.

due to the presence of an increased noise in the link. We tested it with minimum output power at $-18 \mathrm{dBm}$ and 2 Mpbs data rate and a decent transmission quality was up to about $17 \mathrm{~m}$. When the transmitter output power was set to maximum, which is $0 \mathrm{dBm}$, we reached distance somewhat better than $20 \mathrm{~m}$. We also tried with a lower data rate, being set at $250 \mathrm{kbps}$, because at that rate the receiver has a better sensitivity, but we did not notice improvement in the transmission quality at the same distance. (Since it is about a voice transmission, higher nominal data rate and wider bandwidth are a choice in this scenario.) Possible reasons for the noise are multiple: the components used for this prototype were generally low-cost components; the transceivers in use were from different vendors (i.e., have a different quality); there were quite a few wire contacts within the breadboards and between the components, which altogether provide substantial possibilities for noise.

When we replaced a lower-quality transceiver with a higher quality one (Figure 6(a)), we immediately experienced transmission range extension to minimum of $30 \mathrm{~m}$ (Figure 6(b) shows the moment at $15 \mathrm{~m}$ range in a hallway) without losing quality, even with low-gain antennas, which proved one of the above assumptions to be true. (To demonstrate the transmission, an old external speaker was connected in place of a headset and the received sound recorded in a camera video enclosed as a supplementary file (available here).) Both operating channels, i.e., $\mathrm{CH} 1$ and $\mathrm{CH} 75$, had a comparably successful transmission.

The remaining issues will be addressed in the next stage of the development when, besides the quality of parts in use, some connections can be hardwired instead of using a breadboard as an interface. In addition, the number of active components might ultimately be reduced by half using some new approaches.

\section{Real-Time Body Temperature Measurement and Online Publishing}

In this part, we designed a body temperature measuring circuit that submits the measured data to a web page in real time. The emphasis was put on making the circuit reasonably small and getting the real-time data reading at a remote location, such as reading the temperature data off of a designated website from any mobile device. 


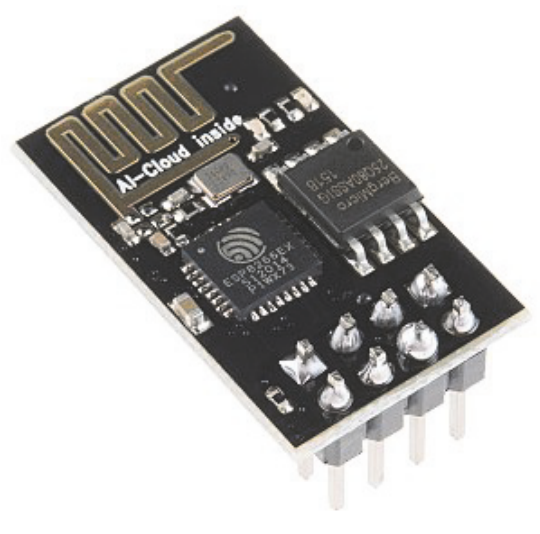

(a) ESP8266

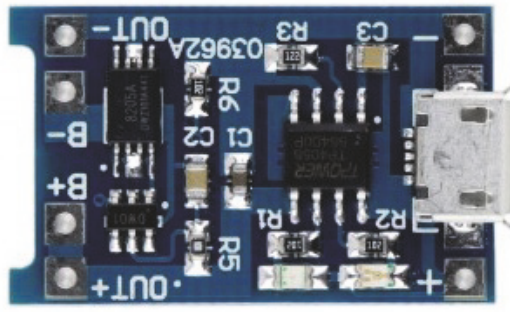

(c) Battery charging module

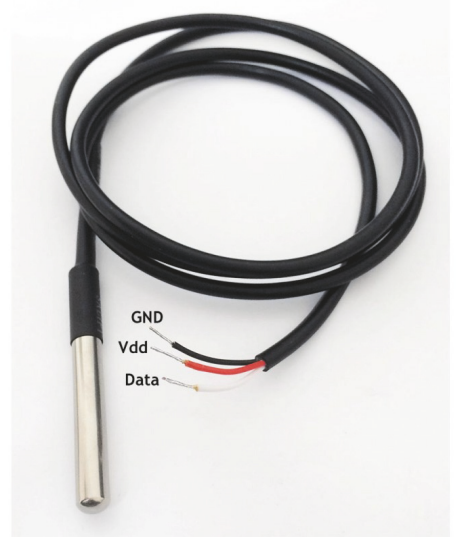

(b) DS18B20 temp. sensor

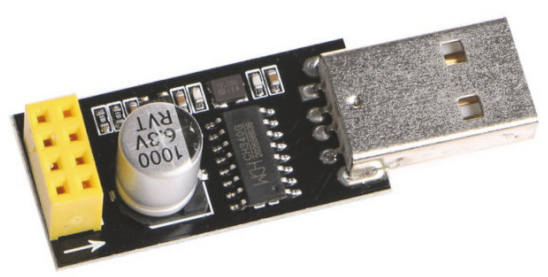

(d) USB-to-ESP8266 adapter

FIGURE 7: Key components that were used to build a real-time published temperature measurement.

The circuit comprises an ESP8266 module [27], DS18B20 temperature sensor [28], a $9800 \mathrm{mAh} \mathrm{Li-ion} \mathrm{battery,} \mathrm{a}$ TP4056 lithium battery charging board [29], and a 5 V-to-3.3 $\mathrm{V}$ step-down DC voltage converter [30] to lower the battery output voltage, which can go up to $4.2 \mathrm{~V}$, and only $3.3 \mathrm{~V}$ is needed for the ESP8266 module. Lastly, in order for ESP8266 module to be programmed, a USB-to-ESP8266 adapter is needed in combination with the drivers for chip 340 [31]. Most of these key components are presented in Figure 7. ESP8266 module is sized only $24.8 \mathrm{~mm}$ by $14.3 \mathrm{~mm}$, which is even smaller than an Arduino nano board. It is similar to Arduino boards for being programmed in the same fashion and the advantage of it is to come equipped with a built-in WiFi module, which is not the case with Arduino boards. On the other hand, an ESP8266 module comes with fewer pins than typical Arduino modules. Unlike Arduino that is biased at $5 \mathrm{~V}$, ESP8266 uses $3.3 \mathrm{~V}$ voltage, which is why it requires a voltage step-down converter from $5 \mathrm{~V}$ to $3.3 \mathrm{~V}$, as mentioned earlier. ESP can work in two modes: as a wireless access point (AP) and as a mobile client that connects to an outside router. The specific URL address is either a fixed URL given by the ESP, if ESP is used as an AP, or given by a router that the ESP module connects to.

DS18B20 temperature sensor can measure temperature with the resolution from 9 to 12 bits, which covers the temperature range from $-55^{\circ} \mathrm{C}$ to $+125^{\circ} \mathrm{C}$. The measured temperature is being converted into a 12-bit word within $750 \mathrm{~ms}$. Each sensor comes with a 64-bit ID, to be uniquely distinguished from other possible sensors in a network and for data communication uses only one wire (i.e., needs only one pin of MCU board).

A lithium battery charging module TP4056 contains an overcurrent protection to preserve the battery from potentially too high a current during charging.

The assembled circuit is shown in Figure 8. The programming adapter shown in the lower half of the carry-on box in Figure 8(a) is superfluous for the run-time operation of the circuit, as it serves only during the programming stage. It is shown here merely as an indication of all the components needed to prepare the solution. As can be seen, the entire circuit, together with the battery, is enclosed within a chassis of $122 \mathrm{~mm}$ by $70 \mathrm{~mm}$, having an outside switch that starts the circuit (Figure 8(b)). The components inside the chassis were glued to keep them safely in place during a motion. When the circuit is running, it periodically submits the temperature reading to the specified URL address, as shown in Figure 9, which was taken off of one mobile phone screen (the specific URL is hidden for this publication).

We also tested duration of the battery discharge cycle to examine how long a fully charged battery can support this circuit. The data are graphically shown in Figure 10. The data show that, after about 250 minutes, the battery dropped from the nominal $4 \mathrm{~V}$ to $3.85 \mathrm{~V}$, which is a drop of about only $4 \%$.

\section{Analysis and Future Work}

The circuits presented here were created to examine the potential of using Arduino microcontrollers as the basis for 


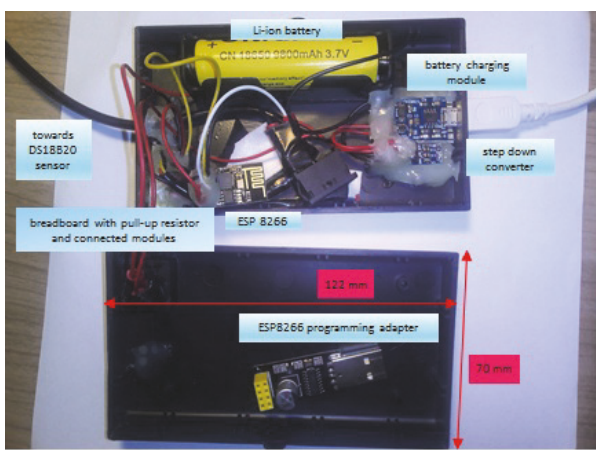

(a) Assembled temperature sensor circuit

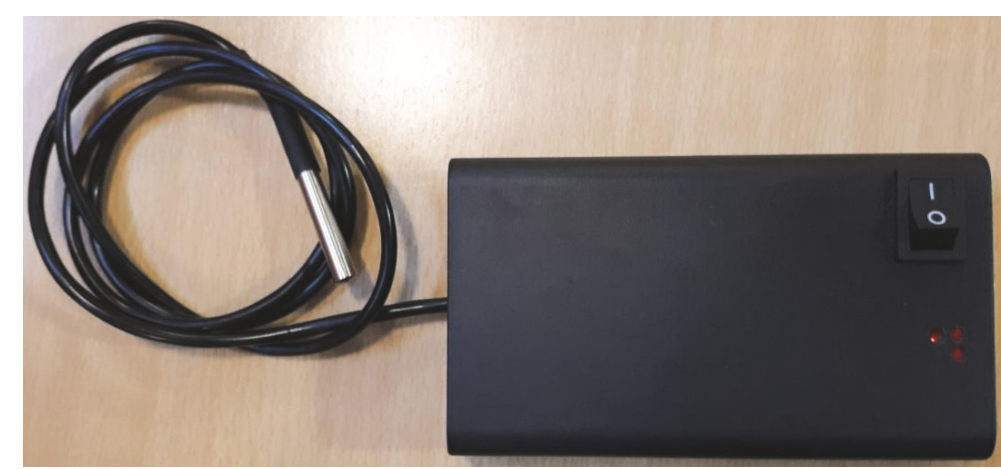

(b) Temperature sensor circuit within a carry-on chassis

FIGURE 8: An early standalone circuit of a temperature sensor that is capable of real-time data publishing: (a) assembled circuit; (b) the circuit within a carry-on box.

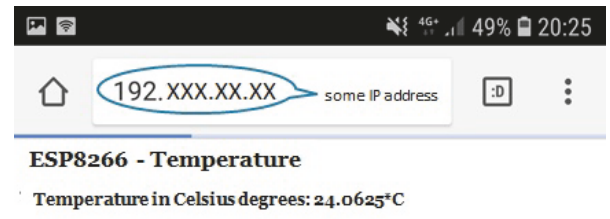

FIGURE 9: A measured temperature is periodically published on the designated web page in real time.

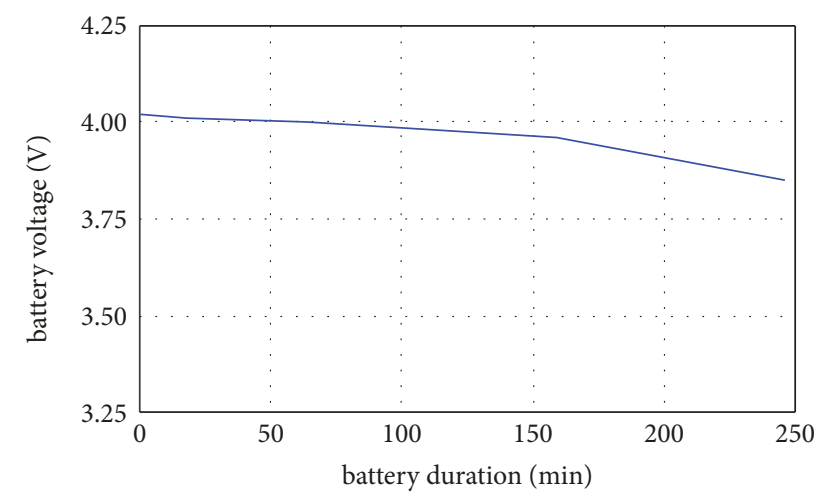

FIGURE 10: Temperature sensor battery discharge cycle measured for the first 250 minutes.

the smart-jacket-embedded electronics and to experience the overall complexity and dimensions of such circuits.

The beneficial aspects of this approach are the low cost and availability of these electronic components, the time to build the circuit (once the idea is mature), and acceptably small dimensions of the key components.

The challenge is a relatively large number of electric contacts that ought to be made to connect all the necessary nodes in the circuit, the number of separate chips that must be connected, the placement and size of the necessary power supply, and the number and placement of the antennas to realize a full-duplex voice communication.

To advance the circuits, the following modified concepts will be examined: (a) getting transceivers more integrated with the Arduino board; (b) exploring if the transceivers and sensors could work together without Arduino boards; (c) using antennas integrated on the transceiver module without losing an option of choosing the operating frequency different from the one in typical commercial circuits; (d) using one antenna for both $\mathrm{Tx}$ and $\mathrm{Rx}$ (instead of two antennas) either by making use of a circulator circuit or by a more sophisticated MCU code that would ultimately make use of a single transceiver for both $\mathrm{Rx}$ and $\mathrm{Tx}$; (e) having conventional batteries replaced with an energy harvestingbased concept [32-35].

It is also to be tested whether antennas that would be integrated within a garment could make a reliable communication link, which would substantially help to avoid cabling between the external antenna and the transceiver, or they will ultimately have to be mounted on the outer surface of the jacket. In any case, it would help to have two transceivers realized on a single chip and have them work at an arbitrary frequency instead of the populated ISM frequency bands. Furthermore, an energy harvesting-based circuit would eliminate the need for replacing the batteries or having the jacket unready due to the charging time, but the question is whether such a circuit would ultimately make the power supply block any smaller than in case when conventional batteries are utilized.

\section{Conclusion}

In this paper, we presented two concept circuits for an envisioned smart jacket, which would be capable of both monitoring a wearer's vital signs and mobile phone-independent full-duplex voice communication. We demonstrated that, even with low-cost off-the-shelf modules and breadboardlevel prototype, it was possible to transfer voice to at least several tens of meters in an open space, without using a Bluetooth connection towards a user's mobile phone, but rather independently on a peer-to-peer basis via a chosen radio frequency. The next version will aim to reduce the number of circuit wire connections, where applicable, make firmer electrical connections in the circuit nodes, replace the job of two transceivers with only one transceiver, and 
test the RF link success based on an external antenna vs. an on-chip antenna, making transceivers operating at an arbitrary frequency, not necessarily at the most populated ISM frequency bands.

In addition, we showed that sensor readings could be transferred to a designated URL address for a real-time viewing from any Internet-connected device. The sensor module is currently based on its connection to a nearby wireless access point, either a router or a hot spot of a mobile phone, while in the future, sensor connection to module serving as an access point itself or having a direct connection to an LTE or $5 \mathrm{G}$ network will be explored for an extended mobility.

An ultimate success will be subject to achieving the smallest possible circuit form factor, while retaining communication quality by means of low noise and bit-error-rate, having a sufficiently long voice communication range at a reasonably low transmitting signal power, all embedded in a way that is noninvasive, comfortable to wear, mechanically robust, and practical to wash or remove before washing. In spite of the technological advancements in all of the aforementioned areas, making a mass production viable product that will fulfill all of the specified requirements is still a challenge.

\section{Data Availability}

The microcontroller codes used to support the findings of this study as well as the videos showing successful voice reception are included within the supplementary information files.

\section{Conflicts of Interest}

The authors declare that they have no conflicts of interest.

\section{Acknowledgments}

This work has been supported in part by the University of Rijeka under the project number uniri-tehnic-18-264.

\section{Supplementary Materials}

Tx1.ino: the code for transmitter 1. Rx2.ino: the code for receiver 2. Tx2.ino: the code for transmitter 2. Rx1.ino: the code for receiver 1 . testing_5m_range_video.mp4: a video clip while testing the 5 - $\mathrm{m}$ voice transfer range. testing_30m_range video_rotated.mp4: a video clip during the test of the $30-\mathrm{m}$ voice transfer range. (Supplementary Materials)

\section{References}

[1] J. Achten and A. E. Jeukendrup, "Heart rate monitoring: applications and limitations," Sports Medicine, vol. 33, no. 7, pp. 517-538, 2003.

[2] A. Ahtinen, J. Mantyjarvi, and J. Hakkila, "Using heart rate monitors for personal wellnessthe user experience perspective," in Proceedings of the Annual International Conference of the IEEE Engineering in Medicine and Biology Society, pp. 1591-1597, 2008.
[3] S. Coyle, F. Benito-Lopez, T. Radu, K. Lau, and D. Diamond, "Fibers and fabrics for chemical and biological sensing," Research Journal of Textile and Apparel, vol. 14, no. 4, pp. 63-72, 2010.

[4] Y. Ohashi, M. Dogru, and K. Tsubota, "Laboratory findings in tear fluid analysis," Clinica Chimica Acta, vol. 369, no. 1, pp. 17$28,2006$.

[5] C. Chen, K. Driggs Campbell, I. Negi et al., "A new sensor for the assessment of personal exposure to volatile organic compounds," Atmospheric Environment, vol. 54, pp. 679-687, 2012.

[6] Omron, HeartGuide ${ }^{\mathrm{TM}}$ : a wearable, clinically accurate, blood pressure monitor, January 2019, https://omronhealthcare.com/products/heartguide-wearable-blood-pressure-monitor-bp $8000 \mathrm{~m} /$.

[7] J. Peckham, "Best fitness tracker 2018: the top 10 activity bands on the planet," December 2018, https://www.techradar.com/ news/wearables/10-best-fitness-trackers-1277905.

[8] OMsignal, Personalized medical-grade biometric data to achieve optimal health, October 2018, https://omsignal.com/.

[9] Sensoria, Sensoria Artificial Intelligence Sportswear, October 2018, http://www.sensoriafitness.com/.

[10] A. A. Calvo and S. Perugini, "Pointing Devices for Wearable Computers," Advances in Human Computer Interaction, vol. 2014, Article ID 527320, 10 pages, 2014.

[11] S. H. Yoon, K. Huo, and K. Ramani, "Wearable textile input device with multimodal sensing for eyes-free mobile interaction during daily activities," Pervasive and Mobile Computing, vol. 33, pp. 17-31, 2016.

[12] S. Griffiths, "9 new fabrics and technologies changing the clothes you wear," T3 SMARTER LIVING, October 2018, https://www.t3.com/features/9-new-fabrics-and-technologieschanging-the-clothes-you-wear.

[13] M. Latifi, P. Payvandy, and M. Yousefzadeh-Chimeh, "Electroconductive textile yarns," in Technical Textile Yarns, Woodhead Textiles Seris No. 101, pp. 298-326, Woodhead Publishing Limited, 2010.

[14] R. Miles, "E-textiles market, evolving technology, industry analysis, share, global trends, key vendors and forecast to 2022," IDA Report, September 2018.

[15] S. Gorgutsa, V. Bélanger-Garnier, B. Ung et al., "Novel wirelesscommunicating textiles made from multi-material and minimally-invasive fibers," Sensors, vol. 14, no. 10, pp. 19260-19274, 2014.

[16] S. Maiti, D. Das, and K. Sen, "Flexible non-metallic electroconductive textiles," Textile Progress, vol. 49, no. 1, pp. 1-52, 2017.

[17] T. Bashir, Conjugated polymer-based conductive fibers for smart textile applications [Doctoral dissertation], Chalmers University of Technology, Sweden, 2013.

[18] A. Chatterjee and S. Maity, "Electroconductive textiles," in Advanced Textile Engineering Materials, S. ul-Islam and B. Butola, Eds., John Wiley \& Sons, Inc., Hoboken, NJ, USA, 2018.

[19] M. Joler, "Towards a smart jacket: integration of electronics challenge," in Proceedings of the International Conference on Sensor Networks and Signal Processing (SNSP '18), Xi'an, China, October 2018.

[20] M. Joler and M. Boljkovac, "A sleeve-badge circularly polarized textile antenna," IEEE Transactions on Antennas and Propagation, vol. 66, no. 3, pp. 1576-1579, 2018.

[21] Arduino platform, May 2018, https://www.arduino.cc/.

[22] Arduino nano, December 2018, https://store.arduino.cc/arduino-nano. 
[23] SparkFun, SparkFun Transceiver Breakout nRF24L01+, May 2018, https://www.sparkfun.com/products/705.

[24] Nordic Semiconductor, Documentation Library. nRF24L01+ Single Chip 2.4GHz Transceiver: Product Specification v1.0, June 2018, https://www.nordicsemi.com/DocLib?Product=nRF24.

[25] Arduino platform, Arduino SPI Library: A Brief Introduction to the Serial Peripheral Interface (SPI), December 2018, https://www.arduino.cc/en/reference/SPI.

[26] Aaronia, "Spectran HF-6065 V4 spectrum analyzer," June 2018, https://www.aaronia.com/.

[27] Wikipedia, ESP8266, June 2018, https://en.wikipedia.org/wiki/ ESP8266.

[28] Maxim Integrated, DS18B20 temperature sensor datasheet, December 2018, https://datasheets.maximintegrated.com/en/ds/ DS18B20.pdf.

[29] Robotop, TP4056 Lithium battery charging board 1A MicroUSB, December 2018, http://www.robotop.lv/en/zaryadkai-zasshita-batarej/502-tp4056-lithium-battery-charging-board1a.html.

[30] Advanced Monolithic Systems, AMS 1117. 1A Low Dropout Voltage Regulator, December 2018, http://www.advanced-monolithic.com/pdf/ds1117.pdf.

[31] SparkFun, USB to serial chip CH340, December 2018, https:// cdn.sparkfun.com/datasheets/Dev/Arduino/Other/CH340DS1 .PDF.

[32] S. Pan, Z. Yang, P. Chen, J. Deng, H. Li, and H. S. Peng, "Wearable solar cells by stacking textile electrodes," Angewandte Chemie International Edition, vol. 53, no. 24, pp. 6110-6114, 2014.

[33] S. H. Kim, "Harvesting electrical energy from carbon nanotube yarn twist," Science, vol. 357, no. 6353, pp. 773-778, 2017.

[34] R. Cao, X. Pu, X. Du et al., "Screen-Printed Washable Electronic Textiles as Self-Powered Touch/Gesture Tribo-Sensors for Intelligent Human-Machine Interaction," ACS Nano, vol. 12, no. 6, pp. 5190-5196, 2018.

[35] Asian Scientist News, "A Wearable Battery You Can Fold And Stretch," October 2018, https://www.asianscientist.com/2018/ 02/tech/flexible-power-source-supercapacitor. 


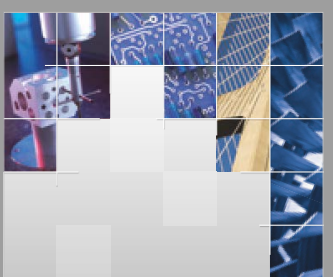

\section{Enfincering}
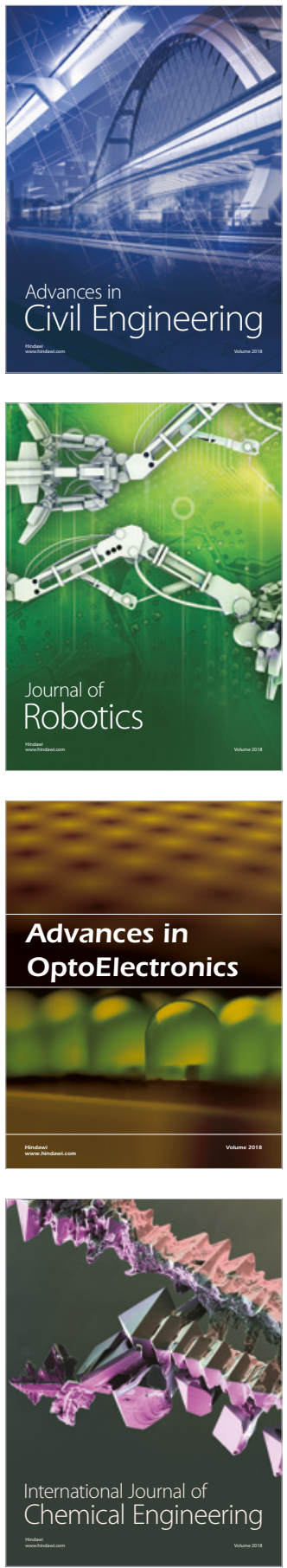

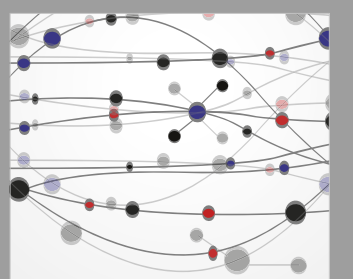

\section{Rotating \\ Machinery}

The Scientific World Journal

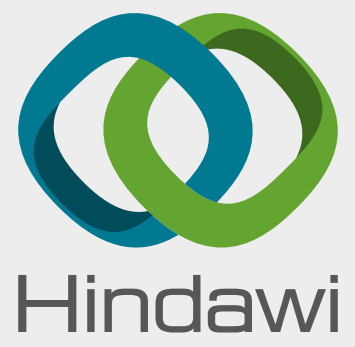

Submit your manuscripts at

www.hindawi.com
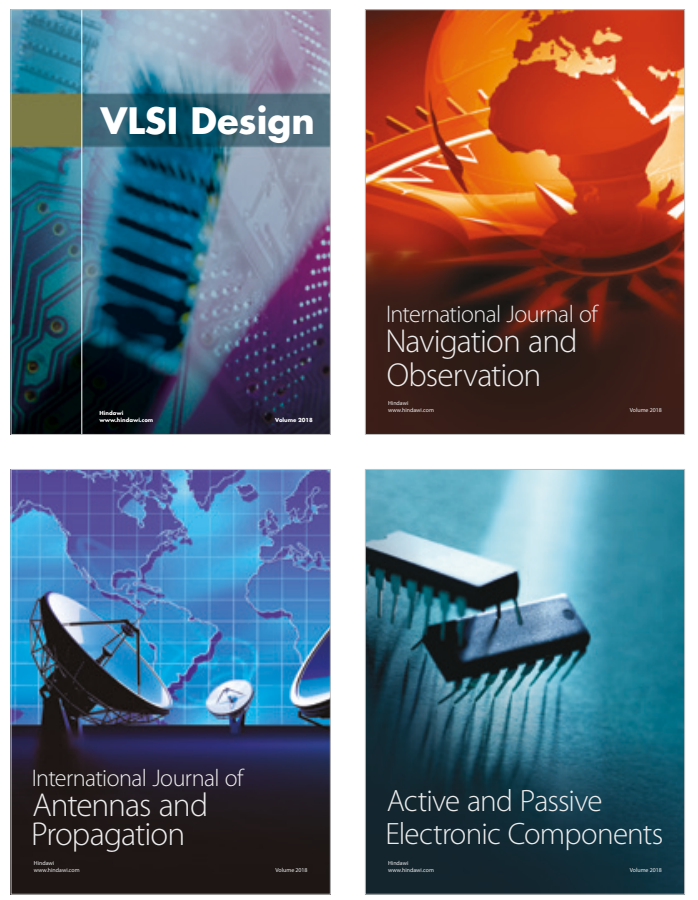
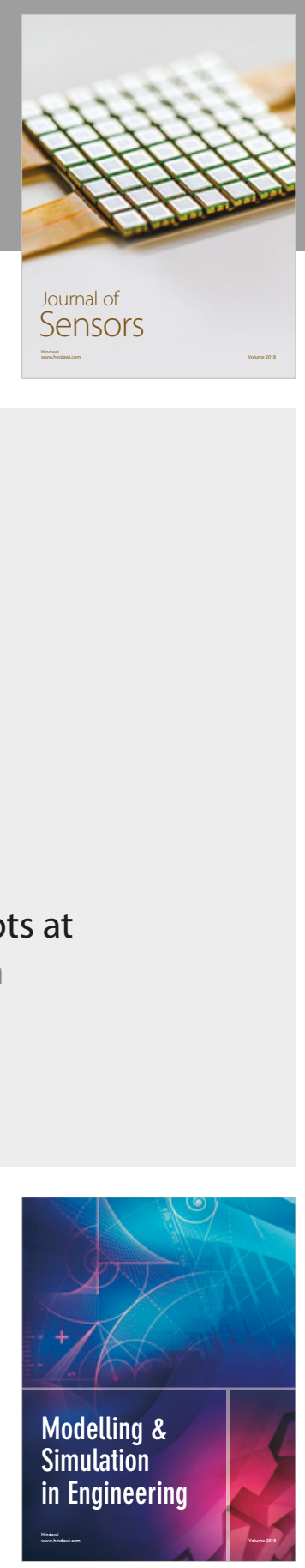

\section{Advances \\ Multimedia}
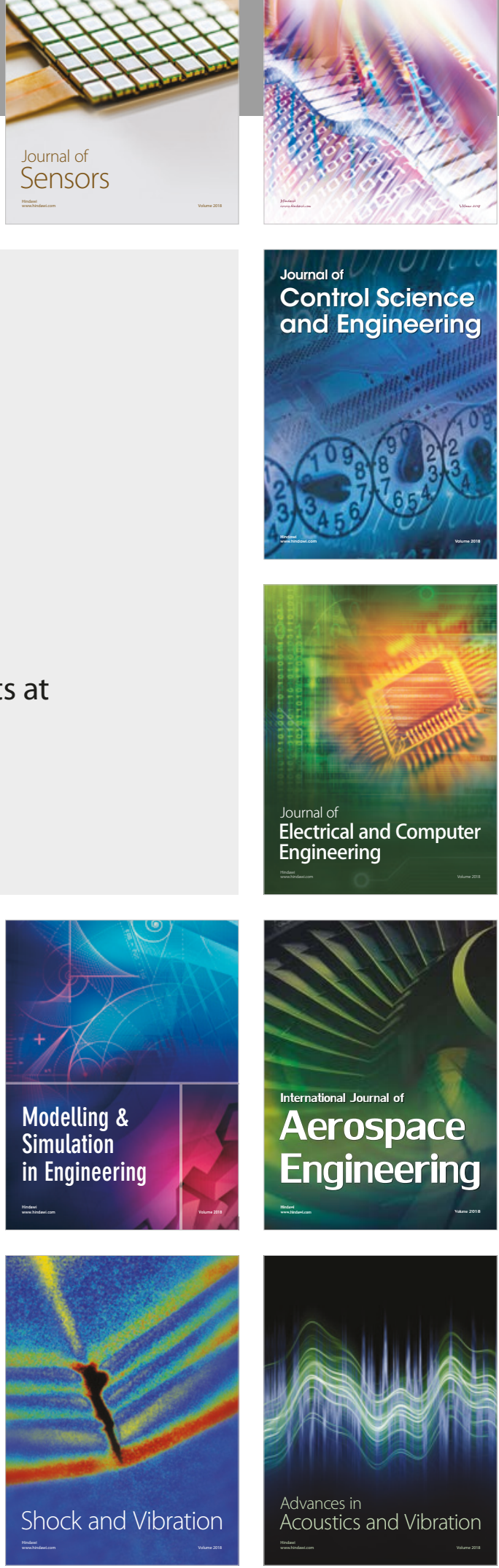